\title{
Extrapolation Theorem on Some Quasi-Banach Spaces
}

\author{
Takuya SOBUKAWA
}

\author{
Okayama University
}

(Communicated by M. Maejima)

Dedicated to Professor Sumiyuki Koizumi on his sixty-fifth birthday

\begin{abstract}
In [5], the author has proved extended sharp extrapolation theorem on $L^{p}$ spaces with $\sum$-method $([1])$, which asserted $\sum_{1<p<q}\left((p-1)^{-\alpha} L^{p}\right)^{p / q}=\left(L \log ^{\alpha} L+L^{q}\right)^{1 / q}$. In the present paper, on that result, we shall consider the case $q \approx 1$.
\end{abstract}

\section{Introduction and result.}

Let $(\Omega, \mu)$ be a $\sigma$-finite measure space. In extrapolation theory on $L^{p}$-spaces, we treat the operator which satisfies the following assumptions.

Assumption. Let $1<q<\infty$ and fix it.

(1) $T$ is a sub-additive operator on $L^{p}(\Omega, \mu)$ for $1<\forall p<q$, i.e. $|T(f+g)| \leq|T f|+$ $|T g|$ a.e. for any $f, g \in L^{p}(\Omega, \mu)$.

(2) For any $f \in L^{p}(\Omega, \mu)$,

$$
\left[\int_{\Omega}|T f(x)|^{p} d \mu(x)\right]^{1 / p} \leq \frac{A}{(p-1)^{\alpha}}\left[\int_{\Omega}|f(x)|^{p} d \mu(x)\right]^{1 / p} .
$$

Here, positive constants $A$ and $\alpha$ are independent of $p$ and $f$.

In real analysis, we can find many operators satisfying such conditions ( $(3)$. In [5], for such operator, the author has proved the following extrapolation theorem:

THEOREM A. Let $T$ satisfy the assumption above. Then, for any $f \in L(\log L)^{\alpha}+$ $L^{q}(\Omega, \mu)$,

$$
\begin{gathered}
\int_{|T f| \leq 1}|T f(x)|^{q} d \mu(x)+\int_{|T f|>1}|T f(x)| d \mu(x) \\
\leq \frac{C_{A}}{(q-1)^{\alpha}}\left[\int_{|f| \leq 1}|f(x)|^{q} d \mu(x)+\int_{|f|>1}|f(x)|(1+\log |f(x)|)^{\alpha} d \mu(x)\right] .
\end{gathered}
$$

Received May 16, 1994 
As a corollary of this result, we can get $\mathrm{S}$. Yano's classical extrapolation theorem:

THEOREM B ([9]). Let $(\Omega, \mu)$ be a finite measure space. Then, for the operator $T$ satisfying the assumption above and the function $f \in L \log ^{\alpha} L$,

$$
\int_{\Omega}|T f(x)| d \mu(x) \leq C_{q, \alpha, A}\left[\int_{\Omega}|f(x)|\left(1+\log ^{+}|f(x)|\right)^{\alpha} d \mu(x)+\mu(\Omega)\right] .
$$

The original proof of Theorem B depends upon the property

$$
L^{1}(\Omega) \supset L^{p}(\Omega) \supset L^{q}(\Omega) \quad \text { for any } \quad 1<p<q .
$$

After the proof of Theorem $\mathrm{B}, \sum_{0<\theta<1} A_{\theta}$, the $\sum$-extrapolation space of strong compatible family of (quasi-)Banach spaces $\left\{A_{\theta}\right\}_{0<\theta<1}$, was defined as

$$
\sum_{0<\theta<1} A_{\theta}=\text { the closure of the linear hull of } \bigcup_{0<\theta<1} A_{\theta}
$$

with the (quasi-)norm

$$
\|a\|_{\Sigma}=\inf \left\{\sum\left\|a_{n}\right\|_{\theta_{n}}: a=\sum a_{n}, a_{n} \in A_{\theta_{n}}\right\} .
$$

It was proved that this space is the "widest" space (see [1, Proposition 2.3]).

For $\alpha \geq 0$, the author proved $\sum_{1<p<q}\left((p-1)^{-\alpha} L^{p}(\Omega)\right)^{p / q}$ is the space of all measurable function $f$ satisfying

$$
\left[\int_{|f| \leq 1}|f(x)|^{q} d \mu(x)+\int_{|f|>1}|f(x)|(1+\log |f(x)|)^{\alpha} d \mu(x)\right]^{1 / q}<\infty
$$

and Theorem A was proved from it. Here, each $\left((p-1)^{-\alpha} L^{p}(\Omega)\right)^{p / q}$ is the space of all $p$-th integrable functions on $(\Omega, \mu)$ with quasi-norm

$$
\left((p-1)^{-\alpha}\|f\|_{\left.L^{p(\Omega, \mu}\right)}\right)^{p / q}=\left[\frac{1}{(p-1)^{p \alpha}} \int_{\Omega}|f(x)|^{p} d \mu(x)\right]^{1 / q} .
$$

However, the original purpose of these studies is the approach to $L^{1}(\Omega)$. As is known, we can never get (1.2) for $q=1$. For example, we shall consider the Hilbert transform $H$. Let $\chi(x)=1$ for $0 \leq x \leq 1$, and $\chi(x)=0$ elsewhere. Then it is easy to show $H \chi \in L^{p}(R)$ for $p>1$ but $\notin L^{1}(R)$. So, instead of the case $q=1$, we shall investigate the following function classes.

Definition (cf. [3]). Let $\alpha, \beta \geq 0 . f \in \mathscr{Z}_{\beta, \alpha}^{*}$ if and only if $f$ is a measurable function on $\Omega$ such that

$$
\int_{|f| \leq 1} \frac{|f(x)|}{(1-\log |f(x)|)^{\beta}} d \mu(x)+\int_{|f|>1}|f(x)|(1+\log |f(x)|)^{\alpha} d \mu(x)<\infty .
$$

Once, the author tried to get some estimation on these classes ([3, Theorem 2]). In this paper, we shall prove the following result, which is the sharpened one. 
THEOREM. Let $(\Omega, \mu)$ be a $\sigma$-finite measure space and let $T$ be an operator satisfying the assumption above. If $f \in \mathscr{Z}_{\varepsilon, \alpha}^{*}$, then $T f \in \mathscr{Z}_{\alpha+\varepsilon, 0}^{*}$ for arbitrary $\varepsilon>0$. Moreover,

$$
\begin{aligned}
& \int_{|T f| \leq 1} \frac{|T f(x)|}{(1-\log |T f(x)|)^{\alpha+\varepsilon}} d \mu(x)+\int_{|T f|>1}|T f(x)| d \mu(x) \\
& \leq C_{q, \alpha, \varepsilon, A}\left[\int_{|f| \leq 1} \frac{|f(x)|}{(1-\log |f(x)|)^{\varepsilon}} d \mu(x)+\int_{|f|>1}|f(x)|(1+\log |f(x)|)^{\alpha} d \mu(x)\right] .
\end{aligned}
$$

Here, the positive constant $C_{q, \alpha, \varepsilon, A}$ depends only on $q, \alpha, \varepsilon$ and $A$.

Moreover, we can treat the spaces $\mathscr{Z}_{\beta, \alpha}^{*}$ as Orlicz spaces. Put

$$
\Phi_{\beta, \alpha}(t)= \begin{cases}\frac{|t|}{(\beta+1)(1-\log |t|)^{\beta}} & (0 \leq|t| \leq 1) \\ \frac{t(1+\log |t|)^{\alpha}}{\alpha+1}+\frac{\alpha-\beta}{(\alpha+1)(\beta+1)} & |t|>1)\end{cases}
$$

and

$$
\Phi_{\beta, \alpha}(L)=\left\{f: \int_{\Omega} \Phi_{\beta, \alpha}(f(x)) d \mu(x)<\infty\right\}
$$

for all $\alpha, \beta \geq 0$. Then, it is easy to show $\Phi_{\beta, \alpha}(L)=\mathscr{Z}_{\beta, \alpha}^{*}$ and each $\Phi_{\beta, \alpha}(L)$ is an Orlicz space with the Luxemberg norm

$$
\|f\|_{\Phi_{\beta, \alpha}(L)}=\inf \left\{\lambda>0: \int_{\Omega} \Phi_{\beta, \alpha}\left(\frac{f(x)}{\lambda}\right) d \mu(x) \leq 1\right\} .
$$

Then we can get

COROLlaRy. Let $(\Omega, \mu)$ be a $\sigma$-finite measure space and let $T$ be an operator satisfying the assumption above. If $f \in \Phi_{\varepsilon, \alpha}(L)$, then $T f \in \Phi_{\alpha+\varepsilon, 0}(L)$ for arbitrary $\varepsilon>0$. Moreover,

$$
\|T f\|_{\Phi_{\alpha+\varepsilon, 0}(L)} \leq C\|f\|_{\Phi_{\varepsilon, \alpha}(L)} .
$$

Here the positive constant $C$ is independent of $f$.

This corollary can be proved similarly to [4, Theorem 1 and 2] and we here omit it.

\section{Proof of the theorem.}

In this section, we shall prove our theorem. First, we shall prove the following lemma.

Lemma. Let $f \in \mathscr{Z}_{\beta, \alpha}^{*}$ and $\beta>0$. Then, 


$$
\sum_{n=1}^{\infty} \frac{1}{n^{1+\beta}} \int_{|f| \leq 1}|f(x)|^{1+(q-1) / n} d \mu(x) \approx \int_{|f| \leq 1}^{q, \beta} \frac{|f(x)|}{(1-\log |f(x)|)^{\beta}} d \mu(x) .
$$

Here, " $X \stackrel{q, \beta}{\approx} Y$ ' means that there exist two positive constants $c_{1}, c_{2}$ which depend only on $q$ and $\beta$ such that $c_{1} Y \leq X \leq c_{2} Y$.

ProOF. It suffices to prove

$$
\sum_{n=1}^{\infty} \frac{1}{n^{1+\beta}} a^{(q-1) / n} \approx \frac{q, \beta}{(1-\log a)^{\beta}}
$$

for any $0<a \leq 1$. First, we consider the case $0<a<e^{-1}$. For $n=1,2,3, \cdots$,

$$
\frac{1}{2^{1+\beta}} \frac{a^{(q-1) / n}}{n^{1+\beta}} \leq \int_{n}^{n+1} \frac{a^{(q-1) / t}}{t^{1+\beta}} d t \leq 2^{1+\beta} \frac{a^{(q-1) /(n+1)}}{(n+1)^{1+\beta}} .
$$

So,

$$
\sum_{n=1}^{\infty} \frac{a^{(q-1) / n}}{n^{1+\beta}} \stackrel{\beta}{\approx} \int_{1}^{\infty} \frac{a^{(q-1) / t}}{t^{1+\beta}} d t=(q-1)^{-\beta}\left(\int_{0}^{-(q-1) \log a} x^{\beta-1} e^{-x} d x\right) \frac{1}{(-\log a)^{\beta}} .
$$

For simplicity, we denote

$$
\Gamma(\beta ; t)=\int_{0}^{t} x^{\beta-1} e^{-x} d x
$$

for any $0<t \leq \infty$. As is known, $\Gamma(\beta ; \infty)=\Gamma(\beta)$. Then, we have

$$
\frac{(q-1)^{-\beta} \Gamma(\beta ; q-1)}{(1-\log a)^{\beta}} \leq \frac{(q-1)^{-\beta} \Gamma(\beta ;-(q-1) \log a)}{(-\log a)^{\beta}} \leq \frac{(q-1)^{-\beta} 2^{\beta} \Gamma(\beta)}{(1-\log a)^{\beta}} .
$$

If $e^{-1}<a \leq 1$, we have

$$
\begin{gathered}
e^{1-q} \sum_{n=1}^{\infty} \frac{1}{n^{1+\beta}} \leq \sum_{n=1}^{\infty} \frac{1}{n^{1+\beta}} a^{(q-1) / n} \leq \sum_{n=1}^{\infty} \frac{1}{n^{1+\beta}} \approx 1+\frac{1}{\beta}, \\
\frac{1}{2}<\frac{1}{(1-\log a)^{\beta}} \leq 1 .
\end{gathered}
$$

Now, we conclude (2.2).

Now, we prove our theorem. From Theorem A, we have

$$
\begin{aligned}
& \int_{|T f| \leq 1}|T f(x)|^{p} d \mu(x)+\int_{|T f|>1}|T f(x)| d \mu(x) \\
\leq & C\left(\frac{1}{p-1}\right)^{\alpha}\left[\int_{|f| \leq 1}|f(x)|^{p} d \mu(x)+\int_{|f|>1}|f(x)|(1+\log |f(x)|)^{\alpha} d \mu(x)\right],
\end{aligned}
$$


for any $1<p \leq q$. Here the constant $C$ depends only on $A$. Put $p=1+(q-1) / n, n \in N$. Multiplying both sides of (2.5) by $n^{-(\alpha+1+\varepsilon)}$ and summing them up with respect to $n$, we get

$$
\begin{aligned}
& \text { (2.6) } \sum_{n=1}^{\infty} \frac{1}{n^{\alpha+1+\varepsilon}} \int_{|T f| \leq 1}|T f(x)|^{1+(q-1) / n} d \mu(x)+\int_{|T f|>1}|T f(x)| d \mu(x) \\
& \leq C_{q, \alpha, \varepsilon, A}\left[\sum_{n=1}^{\infty} \frac{1}{n^{1+\varepsilon}} \int_{|f| \leq 1}|f(x)|^{1+(q-1) / n} d \mu(x)+\int_{|f|>1}|f(x)|(1+\log |f(x)|)^{\alpha} d \mu(x)\right],
\end{aligned}
$$

where the positive constant $C$ depends upon only $q, \alpha, \beta$ and $A$. By using the lemma above,

$$
\begin{aligned}
& \text { the first term of RHS. of }(2.6) \stackrel{q, \varepsilon}{\approx} \int_{|f| \leq 1} \frac{|f(x)|}{(1-\log |f(x)|)^{\varepsilon}} d \mu(x), \\
& \text { the first term of LHS. of }(2.6) \stackrel{q, \alpha, \varepsilon}{\approx} \int_{|T f| \leq 1} \frac{|T f(x)|}{(1-\log |T f(x)|)^{\alpha+\varepsilon}} d \mu(x) .
\end{aligned}
$$

Now, we have completed the proof.

Remark 1. We can never get this theorem for $\varepsilon=0$. For example, $H \chi$, defined in $\S 1$, is not a member of $\mathscr{Z}_{1,0}^{*}(\boldsymbol{R})$.

Remark 2. Assume $q \geq 2$. Multiplying the both sides of (2.5) by $n^{-1-\alpha}(\log (1+n))^{-(1+\delta)}, \delta>0$, we can prove a better estimate

$$
\begin{aligned}
& \int_{|T f| \leq 1} \frac{|T f(x)|}{(1-\log |T f(x)|)^{\alpha}(1+\log \log |T f(x)|)^{1+\delta}} d \mu(x)+\int_{|T f|>1}|T f(x)| d \mu(x) \\
\leq & C_{q, \alpha, \delta, A}\left[\int_{|f| \leq 1} \frac{|f(x)|}{(1+\log \log |f(x)|)^{1+\delta}} d \mu(x)+\int_{|f|>1}|f(x)|(1+\log |f(x)|)^{\alpha} d \mu(x)\right] .
\end{aligned}
$$

Here, $\log \log y=\log (1-\log y)$ for any $0<y \leq 1$.

By using other suitable sequence $x_{n},\left\{x_{n}\right\} \in l^{1}$, we can get similar results.

REMARK 3. T. Miyamoto ([2]) has proved that "If the operator $T$ is of weak-type $(1,1)$ and $(q, q)$ for some $q>1$, then $T$ satisfies $(1.4)$ for $\alpha=1$ ". But our theorem includes his result because the Marcinkiewicz interpolation theorem says "If the operator $T$ is of weak-type $(1,1)$ and $(q, q)$ for some $q>1$, then $T$ satisfies (1.1) for $\alpha=1$ ".

\section{Applications.}

In this section, we state some applications of our theorem.

EXAMPLE 1 (Maximal function). Let $\mathscr{R}_{n}$ denote the collection of all rectangles in $\boldsymbol{R}^{n}$ whose sides are parallel to the coordinate axes for $n \geq 2$ and let $\mathscr{R}_{1}$ denote the collection of a finite open interval in $\boldsymbol{R}^{1}$. For any $f \in L_{l o c}^{1}\left(\boldsymbol{R}^{n}\right)$, we consider the maximal 
function

$$
\mathscr{M} f(x)=\sup _{\substack{R \ni \mathscr{A}_{n} \\ \boldsymbol{R} \ni x}} \frac{1}{|R|}\left|\int_{R} f(x-y) d y\right|
$$

Here, $|R|$ denotes the Lebesgue measure of the set $R$. Then, as is known, the operator $\mathscr{M}$ is of weak-type $(1,1)$ for $n=1$. But for $n \geq 2, \mathscr{M}$ is not of weak-type $(1,1)$ (see $[7$, $\mathrm{X} \mathrm{§2.3]).} \mathrm{Of} \mathrm{course,} \mathrm{even} \mathrm{if} n \geq 2, \mathscr{M}$ is of type $(p, p)$ for $p>1$. Moreover,

$$
\|\mathscr{M} f\|_{L^{p}\left(R^{n}\right)} \leq \frac{A^{n}}{(p-1)^{n}}\|f\|_{L^{p}\left(R^{n}\right)}
$$

(see Remark 3 and [7, II $§ 5.20]$ ). Therefore, instead of the $L^{1}$-boundedness, we have

$$
\|\mathscr{M} f\|_{\Phi_{n+\varepsilon, 0(L)}} \leq C\|f\|_{\Phi_{\varepsilon, n}(L)}
$$

for any $\varepsilon>0$ (see (1.6)). Here, the positive constant $C$ is independent of $f$.

EXAMPLE 2 (Differential operators). Suppose $f$ is of class $C^{2}\left(\boldsymbol{R}^{n}\right)$ and has compact support. Let $\Delta f=\sum_{j=1}^{n} \partial^{2} f / \partial x_{j}^{2}$. Then we have

$$
\left\|\frac{\partial^{2} f}{\partial x_{j} \partial x_{k}}\right\|_{\Phi_{2+\varepsilon, 0(L)}} \leq C\|\Delta f\|_{\Phi_{\varepsilon, 2}(L)}
$$

for any $\varepsilon>0$. Hence, the positive constant $C$ is independent of $f$.

In fact, by using the Riesz transform of the function $f$,

$$
R_{j} f=\frac{\Gamma((n+1) / 2)}{\pi^{(n+1) / 2}} \lim _{\delta \rightarrow 0} \int_{|y|>\delta} \frac{y_{j}}{|y|^{n+1}} f(x-y) d y, \quad j=1,2,3, \cdots, n,
$$

we have

$$
\frac{\partial^{2} f}{\partial x_{j} \partial x_{k}}=-R_{j} R_{k} \Delta f
$$

(see $\left[6\right.$, III §1.3]). As is known, each $R_{j}$ is of weak-type $(1,1)$ and of strong type $(2,2)$. So, as remarked above, $T=R_{j} R_{k}$ satisfies our assumption for $\alpha=2$. Hence we can apply our theorem.

ACKNOWLEDGEMENT. Here, the author would like to express his gratitude to Professor S. Koizumi and S. Ishikawa for their constant encouragement. He also would like to express his thanks to the referee for his suggestion of easier proof of the lemma in $\S 2$.

\section{References}

[ 1 ] B. Jawerth and M. Milman, Extrapolation Theory with Applications, Mem. Amer. Math. Soc. 440 (1991). 
[2] T. MiYамото, On some interpolation theorems of quasi-linear operators (preprint).

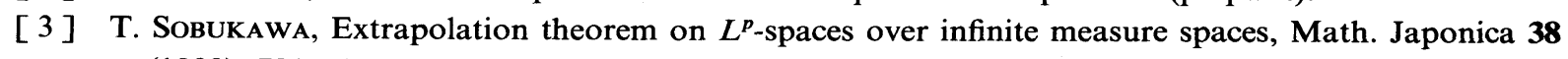
(1993), 781-789.

[4] - Extrapolation theorem and Orlicz spaces, Math. Japonica 41 (1995), 331-338.

[5] Extrapolation theorem on quasi-normed $L^{p}$-spaces, Math. Japonica (to appear).

[6] E. M. STEIN, Singular Integrals and Differentiability Properties of Functions, Princeton Univ. Press (1970).

[7] — Harmonic Analysis: Real-Variable Methods, Orthogonality, and Oscillatory Integrals, Princeton Univ. Press (1993).

[8] T. TAKahashi (=T. KaWATA), On the conjugate function of an integrable function and Fourier series and Fourier transform, Sci. Rep. Tôhoku Univ. 25 (1936), 56-78.

[9] S. YANO, Notes on Fourier analysis (XXIX): an extrapolation theorem, J. Math. Soc. Japan 3 (1951), 296-305.

Present Address:

Department of Mathermatics, Faculty of Education, Okayama University, TSUSHIMANAKA, OKAYAMA, 700 JAPAN.

e-mail: sobu@cc.okayama-u.ac.jp 